\title{
REVISIONES
}

\section{Desafíos de la educación preescolar en Chile: Ampliar la cobertura, mejorar la calidad y evitar el acoplamiento}

\author{
Preschool education challenges in Chile: Enlarge the coverage, \\ improve quality and avoid attach effect
Desafios da educação infantil no Chile: Expandir a cobertura, melhorar a qualidade e evitar o efeito de acoplamento

\author{
Alarcón, Jorge ${ }^{a}$; Castro, Moyra ${ }^{b} ;$ Frites, Claudio $^{c} ;$ Gajardo, Carla $^{d}$ \\ anstituto de Investigación y Desarrollo Educacional (IIDE), \\ Universidad de Talca, Talca, Chile. Teléfono: 56-71-2200253. \\ Correo electrónico: joalarcon@utalca.cl \\ bInstituto de Investigación y Desarrollo Educacional (IIDE), \\ Universidad de Talca, Talca, Chile. Teléfono: 56-71-2200253. \\ Correo electrónico: mocastro@utalca.cl \\ 'Instituto de Investigación y Desarrollo Educacional (IIDE), \\ Universidad de Talca, Talca, Chile. Teléfono: 56-71-2200253. \\ Correo electrónico: cfrites@utalca.cl \\ ${ }^{\mathrm{d}}$ Instituto de Investigación y Desarrollo Educacional (IIDE), \\ Universidad de Talca, Talca, Chile. Teléfono: 56-71-2200253. \\ Correo electrónico: carlagajardo@utalca.cl
}

\section{RESUMEN}

El artículo examina la situación de la educación preescolar en Chile, a consecuencia de la importancia que le conceden algunas iniciativas legales, orientadas al propósito de ampliar su acceso. Se defiende la tesis de que solo efectuando modificaciones sustantivas a las iniciativas propuestas, se evitará replicar en este nivel las reprochables condiciones que aquejan a la educación chilena en su conjunto. El examen presenta y problematiza el contexto en el cual dichas iniciativas han sido propuestas. Vincula la investigación científica, ciertas condiciones políticas y los cambios socio demográficos con el diseño de políticas. Además, expone antecedentes que permiten comprender la situación de la educación preescolar en Chile. Finalmente, ofrece conclusiones que subrayan la necesidad de evitar implicancias negativas en el diseño de políticas de educación preescolar, relevando la necesidad de elaborar políticas comprensivas.

Palabras clave: educación preescolar, acceso, cobertura, políticas educativas, equidad y calidad.

\begin{abstract}
This article assesses the situation of preschool education in Chile as a result of its importance in certain legal initiatives oriented to increase its incorporation and access. The proposal defends that only by applying some fundamental modifications to these initiatives a replication of the detrimental Chilean education conditions can be avoided at this level. The evaluation introduces and questions the context in which these initiatives have been proposed; connects scientific investigations, some political conditions, and the socio-demographic changes with the policies design; and exposes the background which allows the comprehension of the preschool education situation in Chile. Finally, this article presents conclusions in order to emphasise the necessity to avoid negative implications in the policies design for preschool education above the necessity of elaborating comprehensive policies.
\end{abstract}

Key words: preschool education, access, coverage, education policies, equity and quality. 


\section{RESUMO}

Examina-se a situação da educação infantil no Chile, a consequência da importância concedida a ela por algumas iniciativas legais, orientadas para o propósito de ampliar-lhe o acesso. Defende-se a tese de que somente alterando substancialmente as iniciativas propostas evitar-se-á que também se reproduza nessa etapa as censuráveis condições que afligem a educação chilena como um todo. Apresenta-se e problematiza-se o contexto no qual tais iniciativas têm sido propostas. Vincula a investigação científica, certas condições políticas e transformações sociodemográficas ao projeto de políticas. Além disso, expõem-se alguns antecedentes que permitem compreender a situação da educação infantil no Chile. Finalmente, oferecem-se conclusões que evidenciam a necessidade de evitar implicações negativas no projeto de políticas para a educação infantil, destacando-se a necessidade de elaborar políticas inclusivas.

Palavras chave: educação infantil, acesso, cobertura, políticas educativas, equidade e qualidade.

\section{INTRODUCCIÓN}

La educación de la primera infancia en Chile se instala con mayor notoriedad y como objeto de agenda social y política a comienzos del presente siglo teniendo tres coordenadas de acción: el reconocimiento de la educación parvularia como nivel de enseñanza, la universalización de la atención en apoyo a las madres trabajadoras y el fortalecimiento de la iniciativa pública y privada. Siguiendo ese orden, en la actualidad, existe una serie de proyectos legislativos que responden a la gradual atribución de importancia conferida a la educación en los primeros años de vida y a ciertos acontecimientos del país que, unidos a la investigación en este campo, realzan el papel del aprendizaje humano y su vinculación con el desarrollo de las naciones.

En este sentido, baste recordar el discurso del Presidente de la República del 21 de mayo de 2103, anunciando el envío al Congreso de un proyecto de Reforma Constitucional para establecer la obligatoriedad desde los cinco años. Tal modificación, trazada para el último año de educación parvularia, aumenta a trece años la obligatoriedad del sistema escolar y garantiza progresivamente la financiación educacional a los niños de tres años (Gobierno de Chile, 2013a). Coincidentemente, la evidencia empírica indica que la educación en los primeros años de vida contribuye al desempeño escolar futuro, a la superación de inequidades o puede fungir como un importante igualador social (Carneiro \& Heckman, 2003; Dickinson \& Neuman, 2006; Heckman, Stixrud \& Urzúa, 2006). Con un caveat: el potencial igualador de la educación infantil depende de la calidad de su intervención, de lo contrario implica riesgos cognitivos y socioemocionales, particularmente en ambientes sociales empobrecidos (Lira y Contreras, 1999; Bedregal, 2006; Rolla y Rivadeneira, 2006; Bernal \& Keane, 2011; Treviño, Toledo y Gempp, 2013). Desde la esfera política, cada vez se tiende a generar mayor acuerdo en cuanto al impacto de la inversión pública en la educación infantil, debido a las dramáticas cifras de desigualdad en Chile (OCDE, 2011).

La cuestión concerniente a la inversión pública en educación preescolar no se limita, por cierto, al mero incremento de las capacidades individuales para integrarse a la vida laboral (Putnam, 1996). Es un asunto de desarrollo económico y florecimiento humano en situaciones de inequidad (PNUD, 2010) y supone la adopción de un punto de vista acerca de cómo diseñar políticas públicas sobre la base de evidencia científicamente validada. Esta perspectiva es justamente la que motiva el trabajo de Heckman en orden a "vincular esto [la relación entre teorías y datos] a la relevancia y pertinencia práctica para diseñar políticas públicas [...] una visión que nos saca de lo que serían los 
límites tradicionales de las disciplinas e incluso de las políticas públicas tradicionales" (Heckman, 2009: 3).

Las reformas al sistema educativo no han sido atendidas de manera similar en Chile y usualmente han estado asociadas a logros referidos a la (mera) extensión de la cobertura. De hecho, cuales sean los estándares de calidad requeridos por este nivel de enseñanza es un debate postergado o diluido. Ejemplo de ello es el proyecto de ley para autorizar el funcionamiento de jardines infantiles por parte del Ministerio de Educación, el que no se pronuncia sobre estándares vinculados a calidad (Gobierno de Chile, 2013b). Debido a lo mismo, existe cierto escepticismo acerca de cómo evitar que se repliquen en la educación preescolar los problemas que característicamente aquejan al sistema escolar chileno en su conjunto.

El trabajo lleva a cabo sus objetivos regido por una pregunta central, respecto de la que es necesario recabar evidencia que permita responderla, a saber: debido a las transformaciones experimentadas por el sistema educacional chileno desde los años 80 y los efectos negativos que ellas causaron ¿qué tipo de medidas deberían adoptarse en la educación preescolar para que ella produzca igualación social antes que inequidad y segregación?

El artículo aborda estas problemáticas en sus distintas secciones. En la primera de ellas, se examina la importancia de la educación preescolar para el desarrollo individual, sociocultural y productivo. En la segunda sección, por su parte, se muestra evidencia sobre diversas características de la educación preescolar en Chile desde su origen y hasta el presente. En la sección tercera se analiza el nivel educativo, en términos de la evidencia sobre calidad, equidad y eficiencia. Finalmente, el texto ofrece algunas consideraciones sobre la necesidad de adoptar decisiones que respondan a las múltiples dimensiones implicadas en la política educacional sobre la primera infancia.

\section{EL DISEÑO DE POLÍTICAS PARA LA EDUCACIÓN PREESCOLAR}

El debate respecto de la importancia relativa de la educación preescolar en Chile, ha sido influido por un conjunto de hipótesis y resultados de la investigación científica respecto de cuánto se justifica la inversión pública en este nivel. Ello es en parte el resultado de la adopción de nuevos métodos de trabajo y la consecuencia de vincular los resultados de la investigación con el diseño de políticas que favorezcan condiciones de desarrollo de las personas desde la más temprana edad.

En relación con la adopción de nuevos métodos, pueden constatarse sus implicaciones en políticas públicas a consecuencia del enfoque multidisciplinar que nutre el análisis de la evidencia empírica y la visión de conjunto que tal enfoque permite. Así lo señala Heckman (2008) en cuanto al valor que asigna al hecho de integrar en su trabajo a un consorcio de economistas, psicólogos, estadísticos y neurocientíficos, con el objetivo de mostrar la medida en que el desarrollo de la primera infancia influye directamente en la economía, la salud y los resultados sociales para el individuo y la comunidad (Heckman, 2007).

Este último aspecto se hallan particular bien ilustrado en términos de una sistematización de los resultados de la investigación empírica sobre desarrollo y de la propuesta de un modelo de formación de habilidades y destrezas (Human capabilities) que permite, 
precisamente, unificar la evidencia disponible. Los resultados de la investigación son presentados por Heckman $(2007,2008)$ mediante un conjunto de observaciones: las habilidades importan y son de naturaleza múltiple; asimismo, la distinción entre habilidades naturales y no naturales -que ha dominado el pensamiento acerca de la influencia de la familia- ha quedado obsoleta, a causa de carecer de justificación científica. Sobre esta base, Cunha \& Heckman $(2008,2009)$ elaboraron un modelo, algunos de cuyos elementos se sintetizan en lo que sigue (Heckman, 2008).

La brecha de habilidades entre individuos y grupos de distinta condición socioeconómica, lo mismo para habilidades (skills) cognitivas y no $\operatorname{cognitivas}^{1}$, comienza tempranamente, lo que supone intervenir también precozmente para evitar que comience a ampliarse la brecha a favor de aquellas personas que gozan de privilegios sociales y económicos, en desmedro de quienes no gozan de ellos. Tanto en relación con seres humanos como con otras especies animales existe evidencia irrebatible acerca de la existencia de periodos sensibles y críticos en el desarrollo. Este aspecto resulta importante de considerar por cuanto demanda establecer cuándo se requieren apoyos educativos y de otra índole, que apunten a obtener el mayor provecho posible de tales periodos.

La literatura empírica muestra los altos retornos económicos de las intervenciones remediales en niños con desventajas, si estas cumplen con determinados estándares. Los aspectos descritos precedentemente constituyen por ejemplo dos estándares relevantes, es decir, la amplitud de las habilidades consideradas en la intervención y el periodo del desarrollo personal en que se lleve a cabo, pero también el que las inversiones no se limiten a un solo periodo del desarrollo. Asimismo, si la inversión realizada en los primeros años del desarrollo no es seguida por inversiones posteriores, su efecto tiende a menguar. De igual modo, si la inversión no incluye salud y vivienda, entre otros factores que constituyen los apoyos fundamentales para el desarrollo, el impacto tiende a ser cercano a cero. De forma que tanto la integralidad cuanto la continuidad de la inversión es un criterio que debe considerarse como parte de este aspecto. La falta de acceso a créditos tiene un efecto sobre el desempeño que un niño tendrá en el futuro, cuando sea adulto. En este sentido existe evidencia que indica que la falta de crédito durante los primeros años de vida afecta la habilidad de los adultos y su desempeño académico.

Por otra parte, las habilidades nocognitivas fomentan las cognitivas y son un importante predictor del éxito familiar y del éxito de las intervenciones sobre familias con desventajas. La combinación de ambos tipos de habilidades constituye el resultado de la comprensión general del desarrollo humano, en relación con el cual se han realizado significativos avances en los últimos decenios, muchos de los cuales apuntan justamente a la idea surgida contra cierto tipo de política social que suponía la completa separación entre los requerimientos de infraestructura física y aquellos relacionados con el despliegue de competencias individuales.

El modelo de formación de destrezas ${ }^{2}$ propuesto por los mencionados autores considera este conjunto de elementos como base para elaborar una perspectiva que permita

La distinción entre habilidades cognitivas y no-cognitivas se explica mediante la apelación a un elenco de destrezas que las ilustran. Así, por ejemplo, son no-cognitivas o habilidades socioemocionales la perseverancia, la motivación, el autocontrol, etcétera (Heckman, 2008).

2 Al formular su modelo de formación de destrezas, Heckman (2008: 311) señala que se usará skills y ability como sinónimos, y que ambos son el resultado de una combinación entre medioambiente, inversión y genética. 
abarcar la serie de aspectos incidentes en el desarrollo. Al mismo tiempo, la identificación de estos factores permite que el modelo prediga mejor los efectos de las decisiones de política pública sobre el desarrollo individual y sus efectos sobre la comunidad social. A la discusión política y académica se agrega un tercer factor, referido a la evolución de la sociedad y la integración laboral femenina. Al respecto, Pautassi plantea que las políticas sociales desarrolladas por los Estados de Bienestar en Latinoamérica se sustentaron en la conformación de familias con "varones proveedores" y "mujeres amas de casa", en las que la atención de los niños y niñas era sostenida por el trabajo doméstico femenino (Pautassi, 2007:11).

Tal situación ha cambiado por cierto y los cambios resitúan la relevancia de la educación preescolar, desde una perspectiva sociodemográfica, en la medida en que el cuidado de los menores ha salido del espacio familiar y se ha producido una gradual inserción laboral femenina que ha permitido visibilizar una situación antes imperceptible. En este sentido, el Ejecutivo chileno anunció el envío al Congreso Nacional de un Proyecto de Ley que universaliza el derecho a sala cuna de los hijos de mujeres trabajadoras, que en la actualidad se reduce a los hijos de mujeres que se desempeñan laboralmente en empresas con 20 o más trabajadoras en la misma condición (Gobierno de Chile, 2013c). Estos cambios demográficos, sumados a la voluntad política de legislar y la evidencia científica acumulada, justifican la necesidad de una política social que incorpore "el papel fundamental del trabajo de producción y reproducción de las personas, denominado ampliamente como trabajo de cuidado" (Rodríguez, 2012: 27).

\section{CARACTERÍSTICAS DE LA EDUCACIÓN PREESCOLAR EN CHILE}

Sostenidamente, las políticas educativas de la historia republicana de Chile han incrementado el gasto, la cobertura y la obligatoriedad del sistema educacional (Campos, 2000). La educación preescolar no ha sido la excepción: se inicia de manera rudimentaria y aislada durante la segunda mitad del siglo XIX en respuesta al abandono infantil, adquiriendo hoy por hoy un papel preponderante.

En sus orígenes, la educación preescolar estaba asociada al papel de custodia que ejercían ciertas órdenes religiosas de niños y niñas en condiciones de indigencia, abandono $\mathrm{u}$ orfandad, provenientes de las clases populares (Egido, 2000). Posterior a ello, tras el contrato de una maestra extranjera en 1906, se emprende la formación de educadoras y se crea el primer kínder fiscal. El currículum recibe la influencia de la obra de Froebel y más tarde de Montessori y Decroly, definiendo los núcleos centrales de la tarea pedagógica. En 1911 la atención se encauza hacia los menores de sectores populares y desamparados con la fundación del kínder popular, las primeras adaptaciones curriculares al contexto nacional y la extensión de la formación de educadoras en secciones de los liceos de provincias.

En la segunda mitad del siglo XX la carrera profesional para atender educativamente a niños y niñas en edad preescolar es ofrecida por la Universidad de Chile y la práctica se realiza sobre todo en centros poblacionales marginales. El Ministerio de Educación lentamente se incorpora en esta tarea con la elaboración de los programas educativos para el segundo Nivel de Transición y luego al Nivel Sala Cuna. En 1970 con la fundación de la Junta Nacional de Jardines Infantiles (JUNJI) comienza la formación de técnicos 
en educación preescolar en instituciones de enseñanza secundaria técnico-profesional. En 1990 la Fundación Nacional de Ayuda a la Comunidad (FUNACO) se transforma en la Fundación Nacional para el Desarrollo Integral del Menor (INTEGRA), pasando de tener un carácter asistencial a uno educativo. En estos últimos años, la tendencia en educación preescolar es que niños desde sus edades más tempranas participen de alguna experiencia educativa, antes de iniciar la escolaridad obligatoria y formal.

En Chile, la educación formal o regular está organizada en cuatro niveles, entre los que la Educación de Párvulos corresponde al primer nivel de enseñanza reconocido constitucionalmente en virtud de la Ley $\mathrm{N}^{\circ} 19.634 / 1999$. Atiende, cuida y educa integralmente a los niños y niñas en los primeros años vida, parte desde el nacimiento hasta el ingreso al nivel de enseñanza básica. En términos de sus objetivos, intenta favorecer sistemática, oportuna y pertinentemente aprendizajes de calidad para todos los niños y niñas antes del ingreso a la educación obligatoria, lo que plantea en función del bienestar, el desarrollo pleno y la trascendencia de los menores como personas, situación que incluye vínculos y complementariedad con los padres y las familias. La Tabla 1 muestra la distribución de los ciclos y niveles con su correspondiente edad cronológica (MINEDUC, 2012a).

Tabla 1. Ciclos y niveles en educación parvularia

\begin{tabular}{|c|l|l|}
\hline Ciclo & \multicolumn{1}{|c|}{ Nivel } & \multicolumn{1}{c|}{ Edad cronológica } \\
\hline \multirow{3}{*}{$1^{\circ}$} & Sala Cuna Menor & 0 a 11 meses de edad \\
\cline { 2 - 4 } & Sala Cuna Mayor & 1 año a 1 año y 11 meses de edad \\
\cline { 2 - 4 } & Nivel Medio Menor & 2 años a 2 años y 11 meses de edad \\
\cline { 2 - 4 } & Nivel Medio Mayor & 3 años a 3 años y 11 meses de edad \\
\hline \multirow{2}{*}{$2^{\circ}$} & Primer Nivel de Transición & 4 años a 4 años y 11 meses de edad \\
\cline { 2 - 4 } & Segundo Nivel de Transición & 5 años a 5 años y 11 meses de edad \\
\hline
\end{tabular}

Fuente: elaboración propia según el Decreto $N^{\circ} 315 / 2012$. Si es necesario, sala cuna menor puede considerar a menores hasta 1 año 6 meses de edad y sala cuna mayor hasta 2 años 6 meses de edad.

Si sumamos a este marco institucional ciertos antecedentes en torno del financiamiento y la cobertura preescolar, surge un panorama estructural de mayor consistencia respecto de este nivel educacional en Chile, sobre todo si se tienen en consideración los actuales esfuerzos gubernamentales. En consecuencia, la evidencia que se extiende desde 1990 a 2010 presenta aumentos sostenidos en el gasto público y privado respecto de la educación en general, pasando del $4 \%$ al $7 \%$ del gasto del PIB nacional en ambos años. En referencia particular a la educación preescolar, los antecedentes indican que el gasto público sigue siendo mayor al privado al punto que en algunos años el primero triplica al segundo (MINEDUC, 2011). Si se asocia el gasto en educación preescolar con el PIB de Chile, el gasto privado no supera el 0,1\% durante el periodo 2000-2010, mientras que el público aumenta desde 0,2\% a 0,5\% (MINEDUC, 2012b).

Si se examina la cobertura en educación preescolar en Chile, también se percibe un aumento: el comportamiento de la población en edad de asistir a educación de párvulos se ha ido estabilizando en cerca del millón y medio de niños y niñas menores 
de seis años (INE, 2013) y la matrícula en este nivel ha ido aumentando desde los 220.000 alumnos en 1990 hasta los 350.000 en 2010 (Banco Central de Chile, 2012). Las cifras oficiales, provistas por el Ministerio de Planificación Social (CASEN, 19902011), señalan que la cobertura se amplió de $21 \%$ en 1990 a 43,5\% en 2011. Si al comienzo de la década del 2000, 1 de cada 5 niños en la edad correspondiente estaba matriculado, a fines de la misma década prácticamente la mitad de ellos asistía a un establecimiento educacional.

Gráfico 1. Financiamiento público y privado en educación pre-escolar / PIB

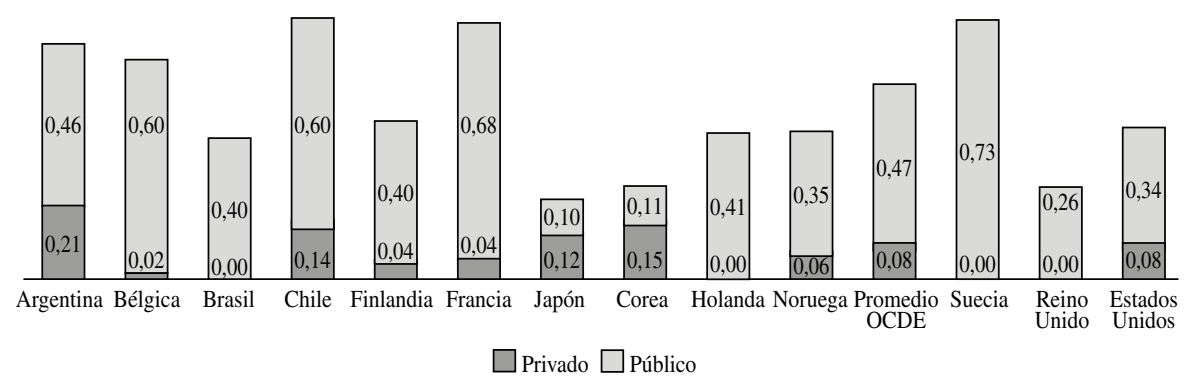

Fuente: OCDE (2011).

Sin contrastes, las cifras apenas informan sobre la situación del financiamiento y la cobertura de la educación preescolar en Chile. Por tanto, en cuanto al gasto público y privado (Gráfico 1) vemos que Chile es uno de los tres países que mayor gasto en educación preescolar presenta como proporción del PIB de toda la muestra $(0,6$ público y 0,1 privado $)^{3}$. En este sentido, solo Francia y Suecia superan a nuestro país y en gasto privado únicamente Argentina.

En la comparación internacional de matrícula preescolar (Gráfico 2), Chile destaca por la tasa de matrícula más baja de los países considerados, con un 55\%, situándose muy por debajo de sus similares regionales, Brasil y Argentina con $65 \%$ y $72 \%$. La mayoría de los países presenta tasas mayores al $80 \%$, siendo Bélgica, Corea, Francia y Suecia los que presentan porcentajes más altos.

En términos de financiamiento, Chile exhibe no solo aumentos sostenidos durante las últimas décadas, sino que también altos índices en términos comparativos. No obstante, la cobertura aún es deficiente, teniendo incluso en consideración el gran salto experimentado desde 1990 a la fecha, puesto que la tasa chilena es la más baja de la muestra considerada. Se entienden, desde esta mirada, los esfuerzos gubernamentales por promover la obligatoriedad, al menos en parte del segundo ciclo del nivel preescolar.

\footnotetext{
3 Los países que conforman la muestra han sido seleccionados combinando diversos criterios: los datos han sido recopilados por la OCDE, algunos son de referencia habitual, en tanto otros constituyen casos ejemplares en equidad y calidad de sus sistemas educativos.
} 


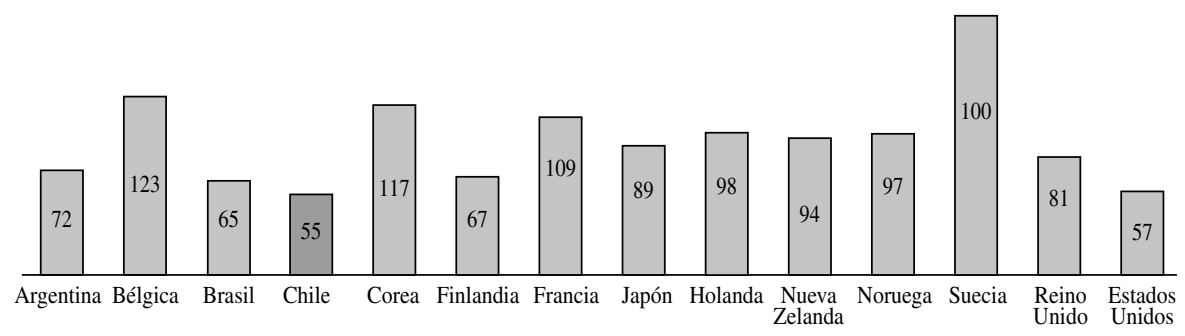

Fuente: OCDE (2011).

\section{CAMBIOS URGENTES: EQUIDAD, CALIDAD Y SEGREGACIÓN SOCIAL}

En relación con los antecedentes expuestos en la sección anterior, se constata cierta concordancia entre las políticas educacionales y las Metas Educativas 2021 que sostienen que es fundamental que los niños y las niñas participen de programas educativos que favorezcan habilidades previas al aprendizaje "y que lo hagan en compañía de otros niños y al cuidado de personal preparado expresamente para este fin" (OEI, 2011: en línea). Dicha concordancia se vincula, además, con la macropolítica pública que promueve el incremento de la productividad y la sustentabilidad y, consecuentemente, la necesidad de valorar la reproducción social y el cuidado (Pautassi, 2007).

La mencionada concordancia es, por otra parte, la que hace evidente la necesidad de adoptar medidas que incrementen la relación entre equidad y calidad en educación preescolar que reduzcan el riesgo de la segregación, efecto respecto del cual el sistema educacional chileno tiene un lamentable récord (Valenzuela, Bellei y De los Ríos, 2010). En este sentido, el impulso a la educación preescolar confluye con políticas para la primera infancia y planes de superación de la pobreza. Esto por cuanto las agendas gubernamentales mostraron los beneficios producidos en el desarrollo de los niños, especialmente en grupos desfavorecidos, mitigando la ausencia y el cuidado parental y determinando el éxito en su etapa de escolarización.

En efecto, la evidencia internacional revela que los programas educativos para preescolares con mayor efectividad son los ejecutados por una educadora profesional que trabaja directamente con los niños (Barnett, 2004; Fukkink \& Lont, 2007). Las investigaciones en distintos jardines infantiles de Chile exhiben un nivel mínimo de calidad, sin progresos notorios en menores de zonas urbanas, particularmente referidos a las competencias cognitivas (Strasser, Lissi y Silva, 2009; Sun, Correa, Zapata y Carrasco, 2011; Lien Fundation, 2012). Muestran, además, limitaciones en cuanto a recintos y materiales de aprendizaje, en tanto que los ambientes educativos son deficientes: si bien las educadoras fortalecen el clima de aula y el vínculo con los niños, son insatisfactorias en mediación de habilidades cognitivas, en el área de lenguaje, del desarrollo socioemocional así como

4 La OCDE elabora la tasa de matrícula con el número de niños matriculados en establecimientos de educación preescolar, siguiendo la clasificación internacional de educación ISCED-0, sin que se correspondan directamente con los sistemas escolares de cada país. De ahí que haya valores superiores al 100\% en la lista de países evaluados. 
también, en cuanto al tiempo efectivo de trabajo pedagógico (MINEDUC, 1998; Villalón, Suzuki, Herrera y Mathiesen, 2002; Álvarez-Salamanca, Díaz y Castro, 2005; Herrera, Mathiesen y Domínguez, 2006; MINEDUC, 2008; Strasser et al., 2009; CEDEP, 2011).

Para fines generales, el Ministerio de Educación es el primer responsable en cuanto al diseño e implementación de políticas, normas legales, criterios de financiación, procesos de fiscalización y supervisión ${ }^{5}$. No obstante, los ciclos señalados en la Tabla I operan de manera aislada y de acuerdo con condiciones de institucionalidad dispar. En razón de ello, legislación, provisión, financiación y fiscalización de los servicios de atención preescolar están asignadas a entidades con lógicas de funcionamiento diferentes (Tokman, 2010). Una muestra de ello es la reciente puesta en marcha del Sistema Nacional de Aseguramiento de la Calidad de la Educación: la Agencia de Calidad evaluará el desempeño de los estudiantes en la Educación Preescolar de los Colegios Municipales y Subvencionados y la Superintendencia de Educación fiscalizará a sus correspondientes sostenedores mediante estándares indicativos convenidos por ley. Así, el desglose y caracterización de establecimientos que imparten educación preescolar se presenta a continuación:

1. Junta Nacional de Jardines Infantiles (JUNJ): cuerpo autónomo de derecho público fundado en 1970 vinculado con el Ministerio de Educación y financiado por el presupuesto anual de la nación. Entrega educación, alimentación y asistencia en forma gratuita y en jornada completa a niños y niñas menores de 6 años, pertenecientes a sectores de escasos recursos de la población.

2. Fundación nacional de atención al menor (Integra): institución educativa de derecho privado sin fines de lucro transformada en 1990, cuya financiación a partir de 2004 procede de la Subsecretaría y Administración General de Educación (Marcel y Tokman, 2005). Atiende gratuitamente a menores desde los 3 meses a los 4 años 11 meses y da prioridad en sus postulaciones a hijos o hijas de familias en situación de riesgo social así como a quienes han sido acogidos en programas de ayudas, de madres trabajadoras, de jefas de hogar cesantes, de padre o madre con discapacidad, de madre adolescente, de niños institucionalizados, entre otros.

3. Jardines infantiles privados: instituciones de derecho privado con fines de lucro (sala cuna y/o jardín) pertenecientes a particulares, empresas u organismos tanto públicos como privados que brindan servicio educativo a niños y niñas hasta los 6 años, cuya financiación es propia o asumida íntegramente por los padres.

4. Educación preescolar en colegios particulares: educación preescolar ofrecida especialmente en el $1^{\text {er }}$ y $2^{\text {do }}$ nivel de transición por medio de proyectos educativos institucionales particulares. Son colegios pagados por los padres y apoderados mensualmente y regulados por contratos de prestación de servicios educacionales. Sin restricción en el monto de las cobranzas, están autorizados para realizar procesos de selección informados, objetivos y transparentes (MINEDUC, 2009).

5. Educación preescolar en colegios municipales: educación impartida en el $1^{\mathrm{er}}$ y $2^{\mathrm{do}}$ nivel de transición que forma parte del proyecto educativo institucional en un establecimiento educacional público administrado por la municipalidad o Corporación Municipal.

Se trata de la Unidad de Educación Preescolar del MINEDUC según la Ley Orgánica de Bases Generales de la Administración del Estado. 
6. Educación preescolar en colegios subvencionados: educación impartida en el $1^{\text {er }}$ y $2^{\text {do }}$ nivel de transición que forma parte del proyecto educativo institucional en un establecimiento educacional particular administrado por personas naturales o jurídicas de carácter privado. El financiamiento deriva del aporte estatal por medio de la subvención de la asistencia del estudiante y, en algunos casos, con los aportes económicos de los padres y apoderados con pagos mensuales. Las normas regulan los montos y los procesos de selección.

En este orden de cosas, la administración está mediada por la dependencia y financiación y, en el plano práctico, la educación preescolar es provista por diversas instituciones de carácter público y privado (Tabla 2). El sistema preescolar mantiene la configuración del sistema educacional en su totalidad. Las prioridades son definidas en el Ministerio de Educación en correspondencia con un modelo mixto que transfiere la administración de centros y la ejecución de programas a organismos de propiedad estatal y particular subvencionada o particular pagada (MINEDUC, 2009).

Si se entiende la equidad de un nivel educacional como la igualdad en términos de cobertura para todos los segmentos socioeconómicos de una sociedad, entonces la equidad en la educación preescolar chilena presenta más bien notorios signos de desigualdad. La cobertura en Chile para este nivel bordea el 55\%, no obstante este parámetro alcanza límites diferentes si se analiza la composición de la matrícula por nivel socioeconómico o dependencia educacional.

Tabla 2. Variantes de Provisión de la Educación Parvularia (EP) ${ }^{6}$

\begin{tabular}{|c|l|l|}
\hline Ciclo & \multicolumn{1}{|c|}{ Nivel } & \multicolumn{1}{c|}{ Provisión } \\
\hline $1^{\mathbf{o}}$ & $\begin{array}{l}\text { Sala cuna menor } \\
\text { Sala cuna mayor } \\
\text { Nivel medio menor } \\
\text { Nivel medio mayor }\end{array}$ & $\begin{array}{l}\text { JUNJI } \\
\text { INTEGRA } \\
\text { Jardines infantiles privados }\end{array}$ \\
\hline $2^{\mathbf{o}}$ & $\begin{array}{l}\text { Primer nivel de tran- } \\
\text { sición (prekínder) } \\
\text { Segundo nivel de } \\
\text { transición (kínder) }\end{array}$ & $\begin{array}{l}\text { JUNJI } \\
\text { INTEGRA } \\
\text { Jardines infantiles privados } \\
\text { EP en colegios particulares } \\
\text { EP en colegios municipales } \\
\text { EP en colegios subvencionados }\end{array}$ \\
\hline
\end{tabular}

Fuente: elaboración propia a partir de datos MINEDUC (2009).

El análisis de datos efectuado a partir de las entregas de la Encuesta de Caracterización Socioeconómica (CASEN) que comprende el periodo de 1990 a 2011 muestra interesantes antecedentes (Gráfico 3) para la población dividida en cinco niveles de ingreso familiar (quintil de ingreso). El primer quintil de ingreso que corresponde a la población más pobre

6 JUNJI e INTEGRA se organizan territorialmente en direcciones regionales con distintas funciones. La primera asume un rol de superintendencia, puesto que supervisa, administra y apoya el trabajo de los jardines infantiles en las regiones. La segunda cuenta con equipos propios para acompañar y supervisar a jardines de su dependencia en las regiones. 
del país presenta niveles de cobertura menores al resto de la población y al promedio nacional: en 1990 la cobertura correspondía a $17 \%$ y a $42 \%$, sin duda un gran avance, pero aún al debe con el promedio de Chile (55\% en 2011). Con el correr de los años se han estrechado las distancias entre cada uno de los estratos analizados; sin embargo, el último quintil de ingreso sigue presentando los niveles más altos de cobertura (58\%).

Gráfico 3. Cobertura preescolar por quintil de ingreso periodo 1990-2011 (\%)

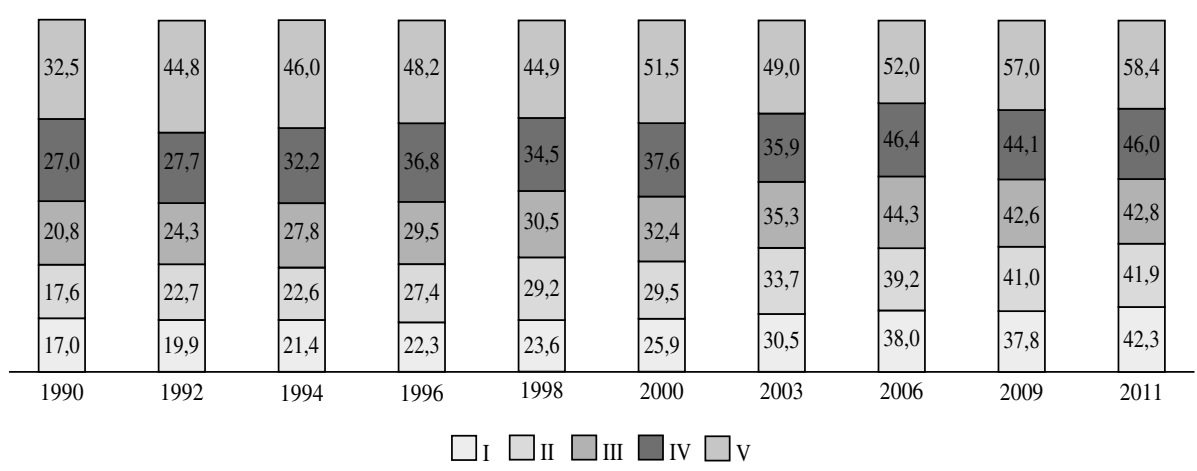

Fuente: elaboración propia a partir de CASEN (1990-2011).

La ponderación de la calidad de la educación preescolar en Chile es una tarea incipiente, que presenta dudas y acumula deudas. La correspondencia con parámetros internacionales, por la falta de información sistematizada y confiable, solo se ha podido hacer desde el cálculo de la tasa alumno/profesor, donde, como era esperable, la posición del país es deficitaria. Países como Finlandia e Irlanda trabajan con menos de 15 niños por profesor. A nivel regional, Chile está por encima de Brasil y Argentina. Las aulas preescolares tienen 25 estudiantes por educadora (MINEDUC, 2011). En cuanto a la medición en relación con parámetros nacionales, el panorama no presenta mejores condiciones y es una discusión abierta que incluye aspectos pedagógicos como la creación de estándares de calidad y los efectos de los resultados para distintos actores (IIPE-UNESCO, 2003). En la actualidad, las discusiones sobre este tópico se han centrado en la acreditación ${ }^{7}$ de las carreras de formación inicial de educadores y en la aplicación de la prueba INICIA ${ }^{8}$.

En relación con acreditación, existiendo 54 carreras de formación profesional para educación preescolar la caracterización según la institución en que se encuentran se desglosa así: el promedio de años de acreditación de las instituciones del Consejo de Rectores de las Universidades Chilenas (CRUCH) supera levemente los cuatro años

La acreditación de la educación superior es un proceso adoptado en Chile que consiste grosso modo en verificar y promover la calidad de la educación, asegurando ciertos mínimos definidos por las universidades y los Estados. Desde 1999 la Comisión Nacional de Acreditación de Pregrado (CNAP) asume el proceso de acreditación, a cuya evaluación se sometían voluntariamente las carreras de pregrado y las universidades. Esta agencia es reemplazada el año 2006 por la Comisión Nacional de Acreditación (CNA), la que actúa por medio de agencias privadas certificadas por la propia CNA.

8 Se trata de pruebas aplicadas voluntariamente a los egresados de todas las carreras de pedagogía y elaboradas en sus distintas etapas por diversas instituciones ligadas al desarrollo educativo, tanto de universidades como de organismos del Estado. 
(43\%), 29\% alcanza 5 años, 14\% alcanza 3 años, 9\% obtuvo 6 años y 5\% no está acreditada; las instituciones privadas consiguen menos años de acreditación en promedio, en efecto, $18 \%$ de las carreras logra 5 años de acreditación, $37 \%$ consigue 4 años, $18 \%$ obtienen 3 años, 9\% apenas 2 años, ninguna obtiene 6 años de acreditación y 18\% simplemente no ha acreditado las carreras. En consecuencia, si se considera la cantidad de años de acreditación como medida de calidad, podría decirse que las carreras impartidas por universidades pertenecientes al CRUCH son mejores que las carreras ofrecidas en universidades privadas, pero es sabido que esta afirmación es cuando menos imprecisa.

En cuanto al segundo indicador, la Prueba INICIA, desde 2009 se aplica la evaluación a los egresados de carreras que imparten educación preescolar. La evaluación a partir de 2012 se divide en conocimientos pedagógicos, disciplinarios y habilidades escritas. Si se desagregan los resultados según el tipo de institución en que estudiaron los evaluados, se tendría que los estudiantes de las instituciones del CRUCH responden incorrectamente poco más de un tercio de las preguntas. Así, los mejores resultados se encuentran en la Pontificia Universidad Católica de Chile y de Valparaíso con un promedio de más del $60 \%$ de respuestas correctas. Los resultados prácticamente se invierten en al menos cuatro universidades (Arturo Prat, de Tarapacá, Los Lagos y Santísima Concepción) con más del $50 \%$ de las respuestas incorrectas. El promedio de respuestas correctas de instituciones privadas muestra resultados heterogéneos: las universidades Central, Diego Portales, Finis Terrae y Los Andes ostentan más del 60\% de respuestas correctas y otras ocho instituciones exhiben más del $50 \%$ de respuestas incorrectas.

Dados los antecedentes, persiste e incluso se acentúa la necesidad de plantear la cuestión acerca de cómo asegurar que la educación preescolar no reproduzca las mismas condiciones de desigualdad del sistema educativo general. El análisis de la cobertura según dependencia y quintil de ingreso demuestra cómo los estudiantes de menores ingresos tienden a matricularse en instituciones de cierto tipo, mientras que los de mayores ingresos en otros. De forma que las familias que pertenecen a los primeros quintiles presentan mayores porcentajes de niños en establecimientos municipales, Integra y JUNJI, en menor medida en los establecimientos particulares subvencionados y muy pocos, en particulares pagados. El primer quintil matricula $40 \%$ de sus hijos en establecimientos municipales y un $4 \%$ en particulares pagados. Al contrario, en los quintiles de mayor ingreso, $56 \%$ inscribe a sus hijos en instituciones pagadas y 4,6\% en municipales. La desigualdad y segregación social se manifiesta en tantos ámbitos como análisis existen, e intentar reducirlas desde los primeros años de vida es una labor necesaria e imperativa por razones de diverso orden. Se debe mejorar la cobertura, eliminar las brechas entre grupos de distinto ingreso económico y asegurar la calidad de los servicios educacionales.

\section{CONCLUSIONES}

A lo largo de las últimas décadas, Chile ha llevado a cabo esfuerzos significativos respecto de la educación preescolar. El aumento en el gasto público y privado, como también la evolución en la cobertura de este nivel, dan cuenta de ello. Sin embargo, no se ha desarrollado el debate necesario en torno de cuál es el tipo de educación preescolar a que se aspira. En este sentido, no deja de ser una paradoja el reciente anuncio de obligatoriedad en la educación preescolar, puesto que no hace más que consagrar una situación que 
casi se presentaba de hecho en referencia a una cobertura real: según CASEN 2011, la cobertura preescolar para niños de cinco años es del 94\% (CASEN, 2011). Hecho que reafirma que la formulación de esta nueva Ley tiene un alcance más bien exiguo respecto del volumen de población a la que efectivamente afectará.

Las referencias que evalúan la inasistencia a educación preescolar (CASEN, 2011) muestran que en Chile son las familias las que deciden no inscribir a sus hijos en algún establecimiento educacional que imparta este nivel de enseñanza. Se ha determinado que las razones que explican este fenómeno están mayormente ligadas a factores culturales, como la valoración que se le da a la educación preescolar, pero también responde a otras problemáticas como la infraestructura de las escuelas o el tipo de acceso que se tiene a ellas. Si las líneas de acción de las políticas públicas buscan incentivar la voluntad familiar y no formular perspectivas que resuelvan inconvenientes estructurales, el efecto no solo será modesto, sino también deficiente como método que fomente un mejor desarrollo de los futuros concurrentes a educación preescolar.

Es razonable que una baja calidad de la formación docente impacta en la calidad de los ambientes educativos. Los estudios nacionales sobre las instituciones formadoras de educadoras de párvulos revelan deficiencias: bajos requisitos de ingreso que según las directoras de carrera son una debilidad fundamental, dispersión y heterogeneidad en las mallas curriculares, escasa interdisciplinariedad como actualización, distantes de los indicadores internacionales de formación inicial. La formación basada en competencias, la educación de la parentalidad asumida en este nivel de enseñanza y la especialización de las educadoras en ejercicio son temas que requieren mayor atención. Si bien los procesos de renovación curricular generan gran interés de las instituciones, exhiben fragilidad en sus condiciones de funcionamiento: reducidas plantas docentes, escasa investigación y especialización de los académicos.

De hecho, la implementación de estándares orientadores es distinta entre una y otra universidad, dependiendo de los recursos (García-Huidobro, 2006; Rojas, Gorichon, Falabella y Lee, 2008; Valverde, Adlerstein y Novoa, 2010; MINEDUC, 2012a). Las educadoras en servicio reconocen debilidades de inclusión y participación en los procesos de gestión institucional, en el rol que cumplen y en las estructuras que atañen a sus objetivos de aprendizaje como la metodología para sus logros. Importa resaltar las dificultades para acceder a la información actualizada de todas las profesionales que laboran en el país y que más del $80 \%$ no cuente con especialización (Rodríguez y Silva, 2009; Romo, Simonstein, Peralta y Mayorga, 2009; Santibáñez, 2009).

Se discute además la capacidad de las iniciativas propuestas por el Gobierno en relación con su ineficacia en un nivel del sistema escolar que carece de estándares de calidad que hayan sido consensuados y que sean comparables con la evidencia internacional o, cuando menos, con los estándares nacionales existentes respecto de vivienda y salud. En efecto, cuando se imparte educación parvularia sin estándares de calidad es posible que sus efectos sean nulos en aquellos alumnos que la han recibido.

Lamentablemente, el proyecto de Ley presentado no propone mejorar aquellos factores que restringen la calidad de la educación preescolar que se imparte en Chile. Tal discusión sigue pendiente y es urgente, ya que bajo el supuesto que se consiga una cobertura total y teniendo esta un estatus obligatorio, nada asegura que no se presente la misma situación que aqueja al sistema escolar chileno respecto de sus déficits estructurales, producto de su diseño estructural y la dinámica existente entre la institucionalidad, el financiamiento 
y sus actores. El problema de esta política pública reside en la posibilidad de que en ese contexto pueda asegurar calidad a mediano y largo plazo, y cuáles serían los mecanismos pertinentes para conseguirlos. Si se extrapola la lógica que impera actualmente en el sistema educacional chileno, no sería extraño que pronto surgiera una evaluación de calidad para preescolares, en vez de debatir sobre la excesiva cantidad de alumnos por profesor en aula o la ausencia de certificaciones obligatorias para enseñar en este nivel de enseñanza, ejemplos de factores que son considerados indicadores de calidad.

El presidente Sebastián Piñera señaló en su mensaje del 21 de mayo de 2013: "Esta reforma comienza con la educación preescolar, porque debemos nivelar la cancha desde la más temprana infancia, de forma de compensar las carencias de origen antes que estas se hagan irreversibles" (Gobierno de Chile, 2013b: en línea). Aunque esta idea se ve refrendada por la evidencia empírica (analizada en la segunda sección), el punto crítico es si la inversión realizada en los primeros años no es seguida por acciones similares durante los años siguientes, el efecto de esos esfuerzos tiende a diluirse. Efectivamente, la obligatoriedad de la educación preescolar sigue siendo un tema controvertido incluso para los países de la OCDE: Australia, Holanda, Hungría, Inglaterra, Israel y México la han promulgado, mientras que en el resto de los países, Suecia y Finlandia incluidas, sigue siendo voluntaria.

Si se es realista, la situación del sistema preescolar no tiene por qué ser diferente. Por lo que es dable esperar que existan centros de educación que, tal como hoy, atraigan a los mejores docentes y logren el desarrollo esperado, mientras que otros establecimientos se transformen en guarderías pensadas solo para facilitar la integración laboral femenina.

Debiese promoverse la universalización del acceso, acentuando la necesidad de enriquecer no solo los contextos familiares, sino también los ambientes de aprendizaje. De no ser así, la obligatoriedad será una iniciativa inconducente. La necesidad de profundizar estos esfuerzos exige coordinar y articular esfuerzos, con la finalidad de diversificar las modalidades de educación parvularia para los niños que no están matriculados: ellos podrían recibir atención educativa oportuna, independiente de sus características individuales, familiares o de territorio. Y por supuesto, medidas que avancen en la compleja tarea de definir y asegurar estándares de calidad apropiados.

\section{REFERENCIAS BIBLIOGRÁFICAS}

Álvarez-Salamanca, E., Díaz, C. y Castro, C. (2005). Calidad de los ambientes de aprendizaje en el primer ciclo de la educación parvularia. Boletín de Investigación Educacional, (20), 235-249.

Banco Central de Chile (2012). Indicadores económicos y sociales regionales de Chile, 1980-2010. Recuperado el 12 de diciembre de 2012 desde http://www.bcentral.cl/publicaciones/ estadisticas/informacion-integrada/iei14.htm

Barnett, W. S. (2004). Better teachers, better preschools: Student achievement linked to teacher qualifications. Preschool Policy Matters, (2). Retrieved January 5, 2014 from http://nieer.org/ resources/policybriefs/2.pdf

Bedregal, P. (2006). Eficacia y efectividad en la atención de niños entre 0 y 4 años. En Foco, (79). Recuperado el 5 de enero de 2014 desde http://www.expansiva.cl/media/actividades/ papers_actividades/20062006075200.pdf

Bernal, R. \& Keane, M. (2011). Child care choices and children's cognitive achievement: The case of single mothers. Journal of Labor Economics, vol. 29 (3), 459-512. 
Campos, N. (2000). Historia de la educación obligatoria en Chile 1810-1997. Santiago: Fundación Claude Bernard.

Carneiro, P. \& Heckman, J. (2003). Human capital policy. In J. Heckman, A. Krueger \& A. Friedman (Eds.), Inequality in America: What role for human capital policies? (pp. 77-239). Cambridge: MIT Press.

CASEN (1990-2011). Encuesta de caracterización socioeconómica nacional. Base de datos. Resultados educación. Recuperado el 5 de enero de 2014 desde http://www.ministeriodesarrollosocial.gob.cl/casen/bases_datos.html

CEDEP (2011). Efectividad de la sala cuna de la Junta Nacional de Jardines Infantiles. Estudio longitudinal 2007-2010. Informe final. Santiago: JUNJI.

Cunha, F. \& Heckman, J. (2009). The economics and psychology of inequality and human development. Journal of the European Economic Association, vol. 7 (2), 320-364.

(2008). Formulating, identifying and estimating the technology of cognitive and noncognitive skill formation. The Journal of Human Resources, vol. 43 (4), 738-782.

Dickinson, D. \& Neuman, S. (Eds.). (2006). Handbook of early literacy research (Vol. 2). New York: Guilford Publications.

Egido, I. (2000). La educación inicial en el ámbito internacional: Situación y perspectivas en Iberoamérica y en Europa. Revista Iberoamericana de Educación, (22). Recuperado el 5 de enero de 2014 desde http://www.rieoei.org/rie22a06.htm

Fukkink, R. \& Lont, A. (2007). Does training matter? A meta-analysis and review of caregiver training studies. Early Childhood Research Quarterly, vol. 22 (3), 294-311.

García-Huidobro, J. (2006). Formación inicial de educadoras(es) de párvulos en Chile. En Foco, (80). Recuperado el 5 de enero de 2014 desde http://www.oei.es/inicial/articulos/formacion_inicial_educadores_parvulos_chile.pdf

Gobierno de Chile (2013a). Mensaje de S.E. el Presidente de la República con el que inicia un proyecto de ley que crea la autorización de funcionamiento de jardines infantiles $N^{o}$ 031-361. Recuperado el 26 de mayo de 2013 desde http://www.camara.cl/pley/pdfpley.aspx?prmID=9060\& prmTIPO=INICIATIVA

(2013b). Mensaje a la Nación de S. E. el Presidente de la República: Chile avanza con todos al desarrollo. Recuperado el 11 de junio de 2013 desde http://www.gob.cl/media/2013/05/ Mensaje-Presidencial_2013.pdf

(2013c). Mensaje de S.E. el Presidente de la República con el que inicia un proyecto de reforma constitucional que establece la obligatoriedad del segundo nivel de transición y crea un sistema de financiamiento gratuito desde el nivel medio mayor $N^{o}$ 091-361. Recuperado el 17 de julio de 2013 desde http://www.camara.cl/pley/pdfpley.aspx?prmID=9202\&prmTIPO=INICIATIVA

Heckman, J. (2009). La economía y piscología del desarrollo humano en contextos de inequidad. Conferencia Ceremonia Inaugural del Centro de Políticas Públicas de la Pontificia Universidad Católica de Chile. Santiago, Chile. Recuperado el 11 de mayo de 2013 desde http://politicaspublicas.uc.cl/publicacion/serie-temas-de-la-agenda/ serie-no35-la-economia-y-psicologia-del-desarrollo-humano-en-contextos-de-inequidad/

(2008). Role of income and family influence on child outcomes. Annals of the New York Academy of Sciences, (1136), 307-323.

(2007). The economics, technology and neuroscience of human capability formation. Proceedings of the National Academy of Sciences, vol. 104 (3), 13250-13255.

Stixrud, J. \& Urzúa, S. (2006). The effects of cognitive and noncognitive abilities on labor market outcomes and social behavior. Journal of Labor Economics, vol. 24 (3), 411-483.

Herrera M., Mathiesen, M. y Domínguez, P. (2006). Evaluación de entornos educativos en centros parvularios para menores de tres años: La escala ITERS. Investigaciones en Educación, vol. 4 (1), 107-127. 
IIPE-UNESCO (2003). Evaluar las evaluaciones. Una mirada política acerca de las evaluaciones de la calidad educativa. Buenos Aires: Instituto Internacional de Planeamiento de la Educación - Organización de las Naciones Unidas para la Educación, la Ciencia y la Cultura.

INE (2013). Sistema de demografía y estadísticas vitales. Santiago: Instituto Nacional de Estadística. Recuperado el 11 de mayo de 2013 desde http://www.ine.cl/canales/chile_estadistico/ familias/demograficas_vitales.php

Lien Foundation (2012). Starting well: Benchmarking early education across the world. Retrieved January 5, 2014 from http://www.economistinsights.com/sites/default/files/legacy/mgthink/ downloads/Starting\%20Well.pdf

Lira, M. I. y Contreras, Z. (1999). Desarrollo psicomotor en lactantes de nivel socioeconómico bajo a cargo de distintos cuidadores. Boletín de Investigación Educacional, (14), 236-246.

Marcel, M. y Tokman, C. (2005). Cómo se financia la educación en Chile. Santiago: MINEDUC.

MINEDUC (2012a). Estándares orientadores para carreras de educación parvularia. Estándares pedagógicos y disciplinarios. Santiago: Ministerio de Educación - Centro de Investigación Avanzada en Educación, Universidad de Chile.

(2012b). Compendio estadístico. Gestión financiera sector público. Matrícula y gasto en subvenciones por nivel. Anuario estadístico 2000 a 2010 (JUNJI e INTEGRA). Santiago: Instituto Nacional de Estadísticas - Contraloría General de la República - Ministerio de Educación.

(2011). Compendio estadístico Junta Nacional de Jardines Infantiles Junji y Fundación Integra. Santiago: Ministerio de Educación. (2009). Ley $N^{o} 20.370$ que establece la Ley General de Educación. Recuperado el 12 de diciembre de 2011 desde http://www.leychile.cl (2008). Prácticas pedagógicas de calidad. Santiago: Ministerio de Educación Universidad de Concepción.

(1998). Evaluación de programas de educación parvularia en Chile: Resultados y desafíos. Santiago: Ministerio de Educación.

OCDE (2011). Growing income inequality in OECD countries: What drives it and how can policy tackle it? Retrieved October 20, 2013 from http://www.oecd.org/social/soc/47723414.pdf

OEI (2011). Miradas sobre la educación en Iberoamérica. Metas educativas 2021. Madrid: Organización de Estados Iberoamericanos - Organización de las Naciones Unidas - Comisión Económica para América Latina y el Caribe.

Pautassi, L. (2007). El cuidado como cuestión social desde un enfoque de derecho. Mujer y Desarrollo, (87). Recuperado el 5 de enero de 2014 desde http://www.unc.edu.ar/extension-unc/ vinculacion/observatorio-ddhh/PresentacionPautassi.pdf

PNUD (2010). Informe regional sobre desarrollo humano para América Latina y el Caribe 2010. Actuar sobre el futuro: Romper la transmisión intergeneracional de la desigualdad. Costa Rica: Editorama.

Putnam, H. (1996). La objetividad y la distinción ciencia-ética. En A. Sen y M. Nussbaum (Eds.), Calidad de vida (pp. 7-26). México D.F.: Fondo de Cultura Económica.

Rodríguez, P. y Silva, A. (2009). Estudio exploratorio descriptivo de los saberes teóricos y prácticos sobre el desarrollo de la psicomotricidad y motricidad infantil, en educadoras de párvulos de establecimientos educativos municipales del país. Santiago: Ministerio de Educación.

Rodríguez, C. (2012). La cuestión del cuidado. ¿El eslabón perdido del análisis económico? Revista CEPAL, (106). Recuperado el 5 de enero de 2014 desde http://www.eclac.cl/cgi-bin/getProd. asp?xml=/revista/noticias/articuloCEPAL/9/46219/P46219.xml\&xsl=/revista/tpl/p39f.xsl

Rolla, A. y Rivadeneira, M. (2006). ¿Por qué es importante y cómo es una educación preescolar de calidad? En Foco, (76). Recuperado el 5 de enero de 2014 desde http://www.expansiva. cl/publicaciones/en_foco/detalle.tpl?iddocumento=19062006104123

Rojas, M., Gorichon, S., Falabella, A. y Lee, M. (2008). Propuestas de mejoramiento para la formación de profesionales de educación parvularia. Santiago: Universidad Alberto Hurtado. 
Romo, V., Simonstein, S., Peralta, V. y Mayorga, L. (2009). Especialización en la formación inicial y continua de educadores de párvulos en los primeros cuatro años de vida de niños/as. Santiago: Ministerio de Educación - Universidad Central.

Santibáñez, D. (2009). Requerimientos técnicos pedagógicos de los educadores de párvulos en el marco de la Ley SEP. Santiago: Ministerio de Educación - Universidad de Chile.

Strasser, K., Lissi, M. R. y Silva, M. (2009). Gestión del tiempo en 12 salas chilenas de kindergarten: Recreo, colación y algo de instrucción. Psykhe, vol. 18 (1), 85-96.

Sun, Y., Correa, M., Zapata, A. y Carrasco, D. (2011). Resultados: Qué dice la evaluación docente acerca de la enseñanza en Chile. En J. Manzi, R. González e Y. Sun (Eds.), La evaluación docente en Chile (pp. 91-136). Santiago: MIDE UC.

Tokman, A. (2010). Radiografía de la educación parvularia chilena: Desafíos y propuestas. Serie de Políticas Públicas, (5). Recuperado el 5 de enero de 2014 desde http://www. sociedadpoliticaspublicas.cl/archivos/MODULO_I/Panel03Radiografia_a_la_educacion_chilena/ Andrea_Tokman-Educacion_parvularia.pdf

Treviño, E., Toledo, G. y Gempp, R. (2013). Calidad de la educación parvularia: Las prácticas de clase y el camino a la mejora. Revista Pensamiento Educativo, vol. 50 (1), 40-62.

Valenzuela, J. P., Bellei, C. y De los Ríos, D. (2010). Segregación escolar en Chile. En S. Martinic y G. Elacqua (Eds.), ¿Fin de ciclo? Cambios en la gobernanza del sistema educativo (pp. 209-232). Santiago: Pontificia Universidad Católica - Organización de las Naciones Unidas para la Educación, la Ciencia y la Cultura.

Valverde, P., Adlerstein, C. y Novoa, X. (2010). Hacia una práctica docente de calidad en el trabajo con familia: Articulando expectativas de instituciones formadoras, política educativa y las familias de la educación inicial. Santiago: Ministerio de Educación.

Villalón, M., Suzuki, E., Herrera, M. y Mathiesen, M. (2002). Calidad de la educación preescolar chilena desde una perspectiva internacional. International Journal of Early Years Education, vol. 10 (1), 49-59. 
